\title{
Research on Raw Materials and Production Technologies of Fuel Ethanol
}

\author{
Hechen Zhang \\ Newton North High School, Boston, 02460, United States of America
}

Keywords: Fuel ethanol, Bioethanol, Straw fermentation, Ethanol production technologies

\begin{abstract}
The world is facing the problem of petroleum energy shortage. Searching for alternative and renewable energy materials becomes the key to solve the energy problem and improve the environment. As a clean and renewable energy, ethanol enters people's vision. As one of the representatives of renewable liquid fuel, fuel ethanol can supplement fossil fuel resources, and has attracted the attention of all countries. In this paper, the development status of fuel ethanol is introduced; the raw materials and the preparation process are explained; the advantages and disadvantages of using different raw materials are expounded; the theoretical differences of different processes and the difficulty of realizing mass production are explored. The objective factors that make the fuel ethanol preparation industry develop better and faster are analyzed in the paper.
\end{abstract}

\section{Introduction}

With the increasing shortage of petroleum energy, the deterioration of the environment and the aggravation greenhouse effect in the world, fuel ethanol as a renewable energy can be used as a new fuel alternative to reduce oil consumption. Fuel ethanol generally refers to absolute ethanol with a volume concentration of $99.5 \%$, which is a good octane number blending gasoline oxygenator. Since the 1970s, the United States and Brazil have taken the lead in issuing policies to encourage the development of fuel ethanol technology and industry. Knowing the prospects for this energy development, many European Union countries have begun to research and develop this new energy. The main raw materials for fuel ethanol include sweet sorghum, cassava, seaweed, and various kinds of cellulose. Each country is developing suitable and qualified ethanol raw materials according to her different advantages.

\section{Bio-Ethanol Raw Materials}

\subsection{Corn Ethanol}

The main raw material for producing corn ethanol is a special kind of corn specially used for ethanol. After the ethanol-producing enzyme gene is transferred into the maize seed by genetic engineering technology, the maize can produce the required enzyme during the processing. The maize with amylase gene can not only produce amylase automatically, but also reduce the viscosity of material and the dosage of enzyme preparation and acid-base, thus reducing the cost and multiplying the result with half the effort. However, with the increasing demand for corn ethanol, more farmland and food are consumed. Many of the corn is used for ethanol production, reduced corn exports and soaring food prices have exacerbated the global food shortage problem. And then corn is overgrown, wildlife habitats are destroyed, and large quantities of fertilizers will flow into wells beneath farmland, into groundwater and rivers, seriously polluting the water resources on which people live.

\subsection{Sugarcane Ethanol}

The energy balance value of ethanol extracted from sugarcane has an obvious advantage over that extracted from corn. Sugarcane ethanol is produced by direct fermentation of sucrose extracted from sugarcane, while corn ethanol requires the hydrolysis of starch by enzymes or catalysts to be converted into distillable sugars for ethanol extraction, which is an additional production process. 
Therefore, the advantages of low cost of sugarcane ethanol make the rapid development of sugarcane ethanol, and promote the cultivation of Brazil, France, Spain and the United Kingdom and other countries. However, even if sugar cane ethanol planting requires less chemical fertilizer, water pollution can be small, sugarcane planting in large areas will still encroach on agricultural land. To cultivate more arable land, many valuable woodland and grassland will be destroyed, and the soil desertification will be aggravated by the greenhouse effect.

\subsection{Cellulosic Ethanol}

Many countries have accelerated the research of cellulosic fuel ethanol because of the problem of "fighting for land with food, fighting for food with people" and the serious pollution to the environment. The raw materials of cellulose are widely used. Lignocellulose is mainly derived from wheat straw, corn stalk, corn cob pulping, fiber residue, waste paper, wrapping paper and other wastes of paper mills, as well as various algae, sugar-containing cassava, sweet sorghum and other non-grain plants. Crop straw is one of the main biomass energy resources in China, and China is a large country of straw production. In recent years, with the development of rural economy, straw is no longer the only choice for farmer's energy consumption and many surplus straws are abandoned in the field. If this kind of cellulose material can be used as the main raw material of the new generation fuel ethanol, it can not only solve the problem of resource waste and pollution, but also develop a huge energy treasure. Library. Cellulose is widely distributed, low cost of cultivation, will not interfere with food production, but the high dispersion of cellulose ethanol raw materials, high production costs, resulting in cellulose ethanol research and development is still in its infancy. Only after optimizing cellulase and reducing cost can cellulosic ethanol enter the stage of large-scale production and market application.

\section{Cure and Process of Raw Materials of Fuel Ethanol}

\subsection{Preprocess of Cellulose Materials}

Hydrogen bonds exist between cellulose molecules and molecules, with high degree of polymerization and crystallinity, and low reactivity. Lignin and hemicellulose in natural cellulose hinder or even block the contact of cellulose molecules with chemical elements and enzymes, making hydrolysis more difficult. Therefore, cellulose materials need to be pretreated to remove lignin and hemicellulose, eliminate space barriers, reduce degree of polymerization and crystallinity, increase reactivity, so that cellulose can be used efficiently. Pretreatment can use physical, chemical and biological methods.

Physical Method. The aggregate structure of cellulose was destroyed by shearing, extrusion and ball milling, and the crystallinity was reduced.

Chemical Method. The lignin and hemicellulose in the raw material were degraded by acid, alkali, organic solvent or oxidant. Dilute acid hydrolysis pretreatment can remove hemicellulose and part of cellulose easily hydrolyzed in raw materials, and improve the enzyme accessibility of raw materials. Lignin can be removed by alkali pretreatment to improve the enzyme accessibility of raw materials, reduce the crystallinity of cellulose, and is easy to hydrolyze cellulase.

Physical and Chemical Method. It includes steam explosion method, ammonia fiber blasting method and so on. The principle of steam explosion is that lignocellulosic biomass is boiled for a period ranging from 160 degrees Celsius to 260 degrees Celsius. High pressure steam enters the cell wall of lignocellulosic biomass through jealousy dissipation, so that after rapidly lowering the temperature, the high-pressure gas inside the cell wall is released to achieve the purpose of lignocellulosic rupture, and then is introduced into the cell wall for use. Acidic substances hydrolyzed. The ammonia fiber blasting method is like that only adding liquid ammonia which can remove lignin.

Biological Method. The degradation of specific lignin by biodegradable lignin or enzymes is mostly caused by white rot fungi. The biological conditions are mild, low energy consumption and less pollution. Compared with the simple effect, the combination of alcoholics and white rot fungi can 
save electricity. Lignin-degrading enzymes are mainly lipase, but there are some substances in the enzyme treatment solution which inhibit the growth of fermentation bacteria and the production of ethanol.

\subsection{Removal of Fermentation Inhibitors}

Cellulose may undergo chemical reactions during pretreatment to produce substances that are toxic to enzymes or inhibit fermentation. These substances can be removed physically, chemically, or biologically. They can also be prevented by controlling toxins and inhibitors during hydrolysis. The removal of inhibitors can be carried out by adsorption, biological or excessive alkali neutralization. The adsorption method needs selective adsorbent, and the adsorbent can be degenerated and regenerated by ethanol. Biological methods require the use of microorganisms or enzymes that can metabolize toxins or inhibitors, but because enzymes are specific to substrates, they are rarely used. The method of excessive alkali neutralization uses alkaline substance to remove the inhibitor from hydrolysate and to remove the inhibitor from dilute acid hydrolysate.

\section{Hydrolysis and Fermentation Process}

Cellulose is the main substance used to produce ethanol in straw, so the hydrolysis of cellulase is necessary to obtain ethanol. The enzyme molecule of cellulose consists of catalytic domain, Junction Bridge and cellulose binding domain (CBD). Among them, the CBD region also has a destructive effect on the structure of polysaccharides, thus promoting the hydrolysis process. Cellulase production is solid-state fermentation and liquid fermentation. Liquid fermentation is generally used, cellulase is easier to extract, and its production cost can be reduced with the enlargement of the scale. However, due to the induction of cellulase production of solid cellulose, the production cycle is long, the efficiency is low, and the amount of cellulase is large, so the higher production cost of cellulase greatly reduces the biological cost. The economics of quality fuel ethanol.

The raw materials used for ethanol production, such as corn stalk, are mainly composed of hemicellulose, the hydrolysate is xylose-based pentose, and then the pentose is fermented to produce ethanol. Therefore, these two key steps need to be controlled in the fermentation process. There are four main methods to produce ethanol by cellulose fermentation: Separate Hydrolysis and Fermentation (SHF), Simultaneous Scarification and Fermentation (SSF), Simultaneous Scarification Co-fermentation (SSCF) and Consolidated Bioprocessing (CBP).

\subsection{Separate Hydrolysis and Fermentation}

The scarification fermentation is divided into two steps. Cellulose was hydrolyzed to sugar at the optimum temperature of cellulase at $50{ }^{\circ} \mathrm{C}$, and then fermented to ethanol at the optimum temperature of yeast at $30^{\circ} \mathrm{C}$. In this method, the two steps will be carried out separately, so that the two enzymes can play the greatest role under the most suitable conditions, without interference with each other, but also with the drawback that the sugar produced after cellulose hydrolysis will inhibit the reaction of cellulose hydrolysis. As the reaction proceeds, sugar accumulates, so the reaction rate slows down, leading to a reduction in yield.

\subsection{Simultaneous Scarification and Fermentation}

To overcome this feedback inhibition, Gauss proposed in 1976 that cellulose hydrolysis saccharification and ethanol fermentation should be carried out simultaneously in the same reactor. Cellulase hydrolysis should be used as the fermentation resource. Because the cellulase hydrolysis and fermentation saccharification of cellulose are carried out continuously in the same device, the hydrolysate glucose is continuously utilized because of the continuous fermentation of bacteria, which eliminates the feedback inhibition of glucose on cellulase due to the increase of substrate concentration. The one-step fermentation process simplifies the equipment, saves the total production time, improves the production efficiency, but because the two-step reaction cannot be controlled to reach the optimum temperature at the same time, it will also reduce the reaction efficiency to a certain 
extent. The State Key Laboratory of Biochemical Engineering, Institute of Process Engineering, Chinese Academy of Sciences, has developed a parallel system for the preparation of ethanol by cellulose bioconversion. The enzymatic hydrolysate separated the enzyme and enzymatic hydrolysate through the nuclear pore membrane. This not only solves the problem that the temperature of enzymatic hydrolysis is not consistent with the fermentation temperature, but also relieves the inhibition of sugar on enzymatic hydrolysis.

\subsection{Simultaneous Scarification Co-fermentation}

The simultaneous scarification and co-fermentation (SSCF) process utilized the co-fermentation strains of C5 sugar and C6 sugar for enzymatic hydrolysis and simultaneous fermentation, which increased the substrate conversion rate and ethanol yield.

\subsection{Consolidated Bioprocessing}

In the above two ways, saccharification and fermentation process are completed by two different microorganisms or enzymes, the corresponding integrated biotechnology emerged. In theory, the microorganism must be able to produce two enzymes. Therefore, genetic engineering techniques, such as Ingram, are needed to clone the genes that can produce ethanol. When ethanol was fermented with cellulase from fungi, the ethanol produced by cellulose increased by $22 \%$. However, the technology required by this method is too complex to be applied in most production because the cost of alcohol production is greatly increased. Mascoma currently uses the technology in its 500 ton/year pilot plant, which uses yeast and bacteria to complete cellulase production and ethanol fermentation. Because of reducing enzyme production units and greatly reducing production costs, Mascoma and Valero have jointly built twenty million gallons/year commercial fibers. The CBP technology will be used in the vitamin ethanol plant. Deinove, a French company, has developed a strain called Mycoccus mirabilis in cooperation with Tereos. Using CBP technology, cellulose can be directly decomposed into monosaccharides and converted into ethanol. The cost of biofuel production is expected to be reduced by $20 \%-30 \%$.

\section{Concentration and Purification Process of Ethanol}

Traditional distillation is used to purify ethanol from biomass fermentation broth. Since ethanol and water can form public waste, extractive distillation and azeotropic distillation are needed to obtain anhydrous ethanol. This method has high energy consumption. Pervaporation process combines membrane separation and evaporation process, which can greatly reduce energy consumption and improve the economy of biomass to fuel ethanol. It is the most promising separation technology. Other dehydration technologies such as molecular sieve adsorption and so on often need to be combined with distillation.

\section{Future and Prospects of Fuel Ethanol}

\subsection{Do not Fight for Food with People and Do not Fight for Land with Food}

The raw materials for bioethanol production are still mainly food. Once the technical difficulties of cellulosic ethanol are overcome, the problem of "fight for food with people and fight for land with food" will disappear and the environment will be improved unprecedentedly, as cellulose is the most widely distributed and most abundant polysaccharide in nature. More importantly, the production of these crops will not interfere with and endanger food production. And some crops can grow and purify soil in wastewater or heavy metal contaminated soil. Therefore, the development of cellulosic ethanol will become a bright spot in the development of biofuels in the future. In recent years, with the increasing pressure on corn stocks and the increasingly serious problem of air pollution, the voice of expanding the use of fuel ethanol is also rising. 


\subsection{Great Potential for Development}

From the international trend of increasing attention to biofuels, China's future fuel ethanol still has great potential for development. Ethanol industry technicians should timely understand the advanced technology and experience at home and abroad to develop suitable ethanol production process package for China and prepare for the future development of ethanol industry.

\subsection{Diversification of Production Materials}

The main advantage of municipal solid waste as raw material is the concentration of raw materials and low cost. For these resources, the main task is to strengthen the garbage classification and rational utilization, and maximize the conversion to fuel ethanol. In Beijing, the volume of garbage disposal was about 4.38 million tons, and the total daily incineration reached 6520 tons (2.38 million tons). If the incinerated waste is converted into fuel ethanol by a synthetic gas route and $200 \mathrm{~kg}$ ethanol is produced from one ton of waste, 476 thousand tons of ethanol can be produced annually. The existing infrastructure (garbage collection, storage and transportation, and classified pretreatment) can replace $13 \%$ of gasoline consumption of Beijing.

\subsection{Lift Technologies}

The use of corn stalks to produce ethanol is a major international problem in the utilization of renewable resources to solve the problem of liquid fuels. Many countries have carried out this work many years ago and have yet to achieve large-scale industrial production. So far, no economic and efficient pretreatment technology can be applied to corn straw pretreatment. Therefore, new pretreatment technologies should be developed in the future, new cellulases with low cost and high activity and new strains of fermentation should be cultivated, and a new ethanol production process with short process. High efficiency and low energy consumption should be developed to reduce the production cost of ethanol.

\section{References}

[1] Zhang Yuxi. Current Development Situation and Prospect for Bioethanol Raw Material [J]. Modern Chemical Research, 2016(4): 43-44.

[2] Li Zhenyu, Li Dingjie, Huang Gesheng, et al. Insights on current development of fuel ethanol [J]. Chemical Industry and Engineering Progress, 2013, 32(7): 1457-1467.

[3] Guo Xiaoxiao, Wu Guoqing. Technology progress of global fuel ethanol industry [J]. Biotechnology \& Business, 2017(1):95-99.

[4] Li Meiqun, Ma Li, Chen Yongzhong. Research Progress in the Fermentation of Fiber Materials to Produce Alcohol [J]. Liquor-Making Science \& Technology, ,2017(11):97-102.

[5] Study on fuel ethanol by synchronous saccharification and fermentation from straws [J]. Renewable Energy Resources, 2013,31(6):85-89. 